\title{
The clinical utility of contrast enhanced whole- heart coronary MRA with 32-channel coil at 3T scanner in the era of 64 and more-slice CT
}

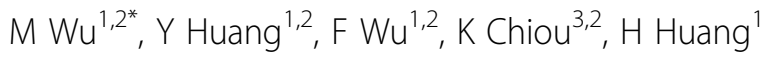 \\ From 16th Annual SCMR Scientific Sessions \\ San Francisco, CA, USA. 31 January - 3 February 2013
}

\section{Background}

Sixty-four and more-slice cardiac CT provides rapid and high quality coronary CT angiography for the majority of clinical needs of non-invasive evaluation of coronary artery. However, the recent advance of whole-heart coronary MRA has been able to provide high-quality of coronary MRA and also myocardial image in a reasonable scan time. The purpose of this study was to evaluate the clinical indication and the feasibility of coronary MRA with 32-channel coil at 3T scanner.

\section{Methods}

Acquisition of 3.0T coronary MRA data was done by using 32-channel torso coil (Skyra, Siemens AG Healthcare, Erlangen, Germany). An ECG-triggered, respiratory navigator-gated, inversion-recovery prepared, segmented gradient-echo sequence was used for image acquisition with an acceleration factor of 3 in the phase-encoding direction using generalized auto calibrating partially parallel acquisitions reconstruction. Slow infusion of 0.15 $\mathrm{mmol} / \mathrm{kg}$ body weight of Gadobenate dimeglumine (MultiHance; Bracco Imaging SpA, Milan, Italy) at a rate of $0.2 \mathrm{~mL} / \mathrm{s}$ was given. The image quality of coronary MRA was scored as 4, excellent; 3, good, confident for diagnosis; 2 . fair, suboptimal for diagnosis; and 1 , poor, not diagnostic. The clinical impact of the examination were classified as high, major revision; medium, minor revision or confirmation and low, non contribution to clinical diagnosis and management.

\section{Results}

Totally 76 patients (median of age $=22$ years, 55 male) received the examination and 71 completed. The 5 failures were due to interrupted anesthesia $(\mathrm{N}=3)$, unstable vital signs $(\mathrm{N}=2)$. The indications of the 71 examination were (1). Kawasaki disease, $\mathrm{N}=26$; (2). anomalous coronary artery, with or without complex congenital heart disease, $\mathrm{N}=8$; (3). assessment of coronary artery disease in young patients with familiar hyperlipidemia, $\mathrm{N}=9$; (4). assessment of coronary artery stenosis in patients with heavy calcification, $\mathrm{N}=11$ and (5). complete evaluation of newly onset heart failure with low risk of coronary artery disease, $\mathrm{N}=17$. The scores of image quality were 18, 42, 8, 3 for scores of 4, 3, 2, and 1 , respectively. The mean image time was $8.2 \mathrm{~min}$. There were additional image findings included: 1 . hyperenhancement of myocardium, $\mathrm{N}=23 ; 2$. myocardial wall hypertrophy, $\mathrm{N}=17 ; 3$. myocardial wall focal thinning, $\mathrm{N}=14 ; 4$. functional valvular disorders, $\mathrm{N}=7$. The clinical impacts of the examination were high in 14, medium in 40 and low in 17 patients.

\section{Conclusions}

Contrast enhanced whole-heart coronary MRA with 32channel coil at $3 \mathrm{~T}$ scanner has high clinical feasibility for appropriate clinical indications. 


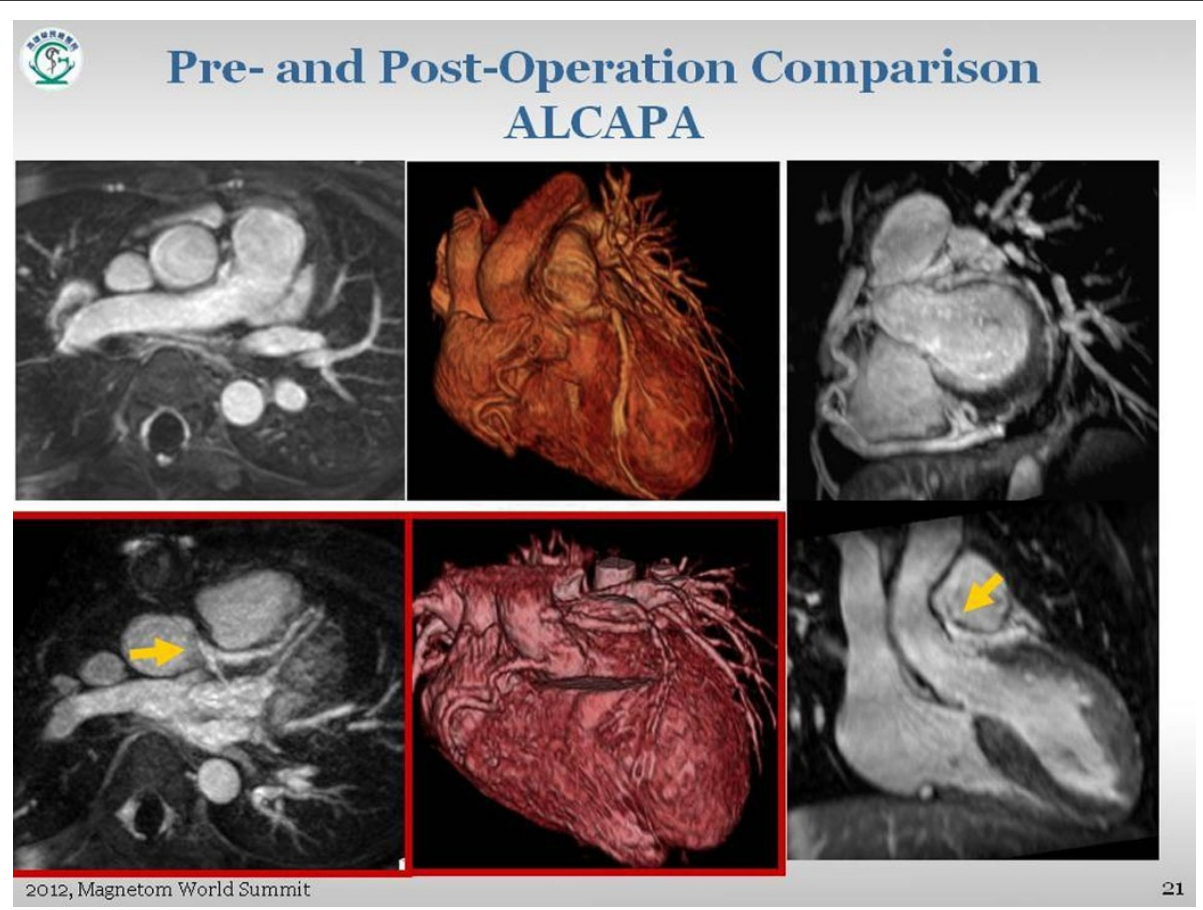

Figure 1 Pre-operation: Aabnormal origin of left coronary artery from the inferior wall of main pulmonary artery, which has patent anastomosis with right coronary artery. Postoperation (lower-right): In-plantation of left coronary artery to the aorta.

\section{Author details}

${ }^{1}$ Radiology, Kaohsiung Veterans General Hospital, Kaohsiung, Taiwan. ${ }^{2}$ Radiology, Faculty of Medicine, School of Medicine, National Yang Ming University, Taipei, Taiwan. ${ }^{3}$ Cardiology, Kaohsiung Veterans General Hospital, Kaohsiung, Taiwan.

Published: 30 January 2013

doi:10.1186/1532-429X-15-S1-W28

Cite this article as: Wu et al:: The clinical utility of contrast enhanced whole-heart coronary MRA with 32-channel coil at 3T scanner in the era of 64 and more-slice CT. Journal of Cardiovascular Magnetic Resonance 2013 15(Suppl 1):W28.

\section{Submit your next manuscript to BioMed Central} and take full advantage of:

- Convenient online submission

- Thorough peer review

- No space constraints or color figure charges

- Immediate publication on acceptance

- Inclusion in PubMed, CAS, Scopus and Google Scholar

- Research which is freely available for redistribution 